\title{
Caracterización de los hogares colombianos en inseguridad alimentaria según calidad de vida
}

\section{Characterising Colombian households regarding quality of life-based food insecurity}

\author{
Martha C. Álvarez-Uribe ${ }^{1}$, Alejandro Estrada-Restrepo ${ }^{1} \mathrm{y}$ \\ Zulma Y. Fonseca-Centeno ${ }^{2}$
}

1 Escuela de Nutrición y Dietética. Universidad de Antioquia. Medellín, Colombia. mcau@pijaos.udea.edu.co, mcalvarez@une.net.co, aestrada@pijaos.udea.edu.co

2 Subdirección de Investigación. Instituto Colombiano de Bienestar Familiar. Bogotá, Colombia. Zulma.Fonseca@icbf.gov.co

Recibido 8 Marzo 2010/Enviado para Modificación 20 Noviembre 2010/Aceptado 5 Diciembre 2010

\section{RESUMEN}

Objetivo Caracterizar los hogares colombianos en inseguridad alimentaria según calidad de vida.

Materiales y métodos Para determinar la calidad de vida de los hogares colombianos se empleó la base de datos de la Encuesta de Calidad de Vida realizada en el año 2008 por el Departamento Nacional de Estadísticas de Colombia. Para establecer las prevalencias de inseguridad alimentaria en el hogar (INSAH) se utilizó la Escala Latinoamericana y Caribeña para la medición de la Seguridad Alimentaria en el Hogar, adaptada y validada para Colombia. Los datos se analizaron con la prueba Chi cuadrado y mediante un análisis de regresión logística.

Resultados En los hogares donde se halló mayor probabilidad de inseguridad alimentaria, fue en aquellos con casa propia pero que la estaban pagando o los que tenían posesión de la vivienda pero sin título, los que manifestaron que los ingresos solo alcanzaban para cubrir los gastos mínimos y en los que no alcanzan para cubrir los gastos mínimos, en los que manifestaron sentirse pobres y en los que vivían hacinados. Perder el empleo por parte del jefe del hogar, atrasarse en el pago de los servicios públicos 4 meses ó más, disminuir el gasto en alimentación y haberse gastado los ahorros, también incremento esta probabilidad.

Conclusión La INSAH se asoció de forma significativa con condiciones socioeconómicas del hogar y del jefe del hogar.

Palabras Clave: Seguridad alimentaria, hambre, calidad de vida, pobreza, Colombia (fuente: DeCS, BIREME).

\section{ABSTRACT}

Objective Characterizing Colombian households regarding quality of life-based food insecurity.

Materials and Methods Colombian households' quality of life was determined using 
data from the quality of life survey carried out by the Colombian Statistics' Department in 2008. The Latin-American and Caribbean Household Food Insecurity Scale was used for measuring the prevalence of household food insecurity(after having been adapted and validated for Colombia). Data was analyzed using the Chi square test and logistic regression analysis.

Results The households having a higher probability of food insecurity were those owning their dwelling but still paying the mortgage or those who were in possession of a dwelling without ownership documents, households where income was deemed insufficient for covering minimum expenses or did not cover minimum expenses, those who stated that they felt poor and those living in overcrowded conditions. The probability of food insecurity was also increased in households where the head of the family had lost her/his job, where late payments of 4 months or more were due for public utilities, had decreased food expenses or had spent their savings.

Conclusion Household food Insecurity was significantly associated with a household's socioeconomic conditions and those of a head of household.

Key Words: Hunger, quality of life, household, poverty, Colombia (source: MeSH, NLM).

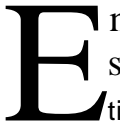

n la Cumbre Mundial sobre Alimentación, celebrada en Roma en 1996 se definió la seguridad alimentaria "cuando toda persona en todo momento tiene acceso económico y físico a suficientes alimentos inocuos y nutritivos para satisfacer sus necesidades alimentarias y preferencias en cuanto alimentos a fin de llevar una vida sana y activa" (1).

El concepto de inseguridad alimentaria y hambre se establece a partir de un panel de expertos convocado en 1989 por el Instituto Estadounidense de la Nutrición para la Organización de la Investigación en Ciencias de la Vida. Hoy se conceptúa como "Inseguridad alimentaria la disponibilidad limitada o incierta de alimentos nutricionalmente adecuados e inocuos o la capacidad limitada e incierta de adquirir alimentos adecuados en formas socialmente aceptables" (2).

Para la medición de la inseguridad alimentaria en el hogar basada en la experiencia, se utiliza una escala compuesta por varios ítems que cubren la preocupación de los hogares porque los alimentos se van a acabar y no se tienen los recursos para adquirirlos, la reducción de la cantidad de alimentos disponibles en el hogar, el deterioro de la calidad de la alimentación y la experiencia de hambre. Algunas preguntas están dirigidas al hogar, otras preguntas se refieren a los adultos y a los niños que habitan en el domicilio $(3,4)$. 
Para la evaluación de la seguridad alimentaria en el hogar mediante escalas cualitativas, se han definido cuatro categorías (5). Seguridad alimentaria: el hogar muestra un mínimo o nada de evidencia de inseguridad alimentaria. Inseguridad alimentaria leve: en esta categoría se evidencia la preocupación de los miembros del hogar por el abastecimiento suficiente de alimentos y los ajustes alimentarios, como la disminución de la calidad de los alimentos y se reporta poco o ninguna reducción en la cantidad de alimentos que usualmente realiza el hogar. Inseguridad alimentaria moderada: en este grado de inseguridad alimentaria la ingesta de alimentos por los adultos han sido reducidas de tal modo que implica una experiencia sicológica constante de sensación de hambre. Inseguridad alimentaria severa: todos los miembros del hogar han reducido la ingesta de alimentos hasta el extremo en que los niños experimentan hambre.

Los hogares más expuestos a la inseguridad alimentaria son los encabezados por campesinos pobres con producción de alimentos marginal o inadecuada, por mujeres pobres, compuestos por un alto número de personas, los que viven en áreas ecológicamente de riesgo y con ingresos muy bajos $(6,7)$. Entre los factores que se han identificado como determinantes de la seguridad alimentaria y el bienestar nutricional del hogar se encuentran la suficiencia de alimentos en el mercado y otros canales de comercialización de alimentos (8), la estabilidad de los suministros alimentarios (9), la capacidad de los hogares para adquirir los alimentos que ofrece el mercado lo cual depende del ingreso y de los precios de los alimentos, del cuidado adecuado de los integrantes del hogar y en particular a los niños (9).

Este artículo tiene como objetivo caracterizar los hogares colombianos en inseguridad alimentaria según condiciones de vida en el hogar durante el año 2008.

\section{METODOLOGÍA}

Tipo de estudio: Descriptivo transversal.

Encuesta de Calidad de Vida en Colombia

Para caracterizar la inseguridad alimentaria de los hogares colombianos, se utilizó la base de datos de la Encuesta de Calidad de Vida (ECV) en Colombia del año 2008, realizada por el Departamento Nacional de Estadística (DANE), cuyo objetivo fue obtener información que permitiera analizar y realizar comparaciones de las condiciones socioeconómicas de los hogares 
colombianos, pertinentes para hacer seguimiento a las variables necesarias para el diseño e implementación de políticas públicas y para el seguimiento de los Objetivos de Desarrollo del Milenio (ODM)(10). El diseño de la muestra fue probabilístico, estratificado y poseía criterios de estratificación, multietápico de conglomerados (10). La submuestra estuvo constituida por 13.602 hogares de Colombia.

Escala de seguridad alimentaria

Se utilizó la Escala Latinoamericana y Caribeña para la medición de la Seguridad Alimentaria (ELCSA) adaptada y validada en Colombia $(11,12)$. La cual está constituida por 15 Ítems, los 9 primeros se refieren a situaciones relacionadas con los adultos y las restantes a los niños. La opción de respuesta de cada ítem es dicotómica, a la opción Si se le asignó un puntaje de uno [1] y a la opción No de cero [0]. Los puntos de corte utilizados para clasificar la seguridad se presentan en la Tabla 1.

Tabla 1. Puntos de corte para clasificar el grado de Inseguridad Alimentaria (ISA) en el hogar

\begin{tabular}{lcc}
\hline Seguridad alimentaria & Hogares con niños & Hogares sin niños \\
\hline Seguros & 0 & 0 \\
ISA leve & $1-6$ & $1-4$ \\
ISA moderada & $7-11$ & $5-7$ \\
ISA severa & $12-15$ & $8-9$ \\
\hline
\end{tabular}

Análisis estadístico

De la encuesta de calidad de vida se analizaron las siguientes variables. Localización geográfica del hogar: urbana y rural. Características de la vivienda: estrato, tipo, servicios públicos, combustible y lugar para la preparación de alimentos. Características del hogar: hacinamiento, ingresos, pago de servicios públicos, pérdida del empleo, gasto alimentario y endeudamiento. Características del jefe del hogar: sexo, nivel educativo y ocupación. Condiciones de vida: percepción condiciones de vida y pobreza. La asociación de la clasificación de seguridad alimentaria y algunas características sociales y demográficas de los hogares y del jefe de hogar se efectuó con la prueba de Chi cuadrado. Mediante el análisis de regresión logística se calcularon las razones de disparidad (OR) con sus intervalos de confianza al $95 \%$, para determinar la probabilidad de inseguridad alimentaria de los hogares de acuerdo a características económicas, sociales y demográficas de los hogares. El nivel de significancia establecido fue del $5 \%$. Los análisis fueron llevados a cabo en el software SPSS versión 17 (SPSS. Statistical package for the social sciences, version 17 for Windows. Chicago: SPSS Inc., 2008) 


\section{RESULTADOS}

Prevalencia de inseguridad alimentaria en los hogares - ISAH

Según la ECV, en el año 2008 el 58,2 \% de los hogares colombianos se encontraban en inseguridad alimentaria y entre ellos el 37,9\% estaban en inseguridad leve, el $11,6 \%$ en moderada y el 8,7 \% en severa. Los hogares que vivían en el área rural se encontraron en mayor proporción en ISA, con respecto a los que vivían en el área urbana. Esta misma situación se presentó en todos los grados de IS A ( $\mathrm{p}=0,000)$ (Tabla 2$)$.

Tabla 2. Grado de inseguridad alimentaria según características de la vivienda y socioeconómicas del jefe del hogar. Colombia, ECV 2008

\begin{tabular}{|c|c|c|c|c|c|c|}
\hline \multirow[b]{2}{*}{ Características } & \multicolumn{5}{|c|}{ Grado de inseguridad alimentaria (\%) } & \multirow[b]{2}{*}{$\mathrm{p}^{\star \star \star}$} \\
\hline & $\begin{array}{l}\text { Seg } \\
\text { uros }\end{array}$ & Leve & Moderada & Severa & $I S A^{* \star}$ & \\
\hline \multicolumn{7}{|l|}{ Localización geográfica } \\
\hline Colombia & 41,8 & 37,9 & 11,6 & 8,7 & 58,2 & \multirow{3}{*}{0,00} \\
\hline Urbana & 45,6 & 36,5 & 10,1 & 7.7 & 54,3 & \\
\hline Rural & 28,4 & 42,7 & 16,8 & 12,0 & 71,5 & \\
\hline \multicolumn{7}{|l|}{ Estrato socioeconómico } \\
\hline $\begin{array}{l}0 \text { (Factura sin estrato o } \\
\text { pirata) }\end{array}$ & 302 & 269 & & & & \multirow{7}{*}{0,00} \\
\hline 1 (Baio-Baio) & 30,2 & $\begin{array}{l}26,9 \\
41,5\end{array}$ & $\begin{array}{l}19,2 \\
18,8\end{array}$ & 23,7 & 69,8 & \\
\hline 2 (Baio) & 20,0 & $\begin{array}{r}41,5 \\
41,3\end{array}$ & 18,8 & 14,2 & 14,5 & \\
\hline 3 (Medio-Baio) & 565 & 34,8 & 56 & 3,1 & $\begin{array}{l}58,1 \\
435\end{array}$ & \\
\hline 4 (Medio) & 74.6 & 209 & 2.9 & 1.6 & $\begin{array}{l}43,5 \\
25,4\end{array}$ & \\
\hline 5 (Medio-Alto) & 76,4 & 208 & 28 & 0.0 & $\begin{array}{l}20,4 \\
23,6\end{array}$ & \\
\hline 6 (Alto) & 88,2 & 11,8 & 0,0 & 0,0 & 11,8 & \\
\hline \multicolumn{7}{|l|}{ Tipo de vivienda } \\
\hline Casa & 35,1 & 40,1 & 14,5 & 10,3 & 64,9 & \multirow{5}{*}{0,00} \\
\hline Apartamento & 50,3 & 36,2 & 8,4 & 5,1 & 49,7 & \\
\hline Cuarto inquilinato & 39,3 & 33,3 & 11,0 & 16,4 & 60,7 & \\
\hline Cuarto otro tipo* & 34,8 & 33,4 & 15,5 & 16,2 & 65,1 & \\
\hline Otro tipo de vivienda & 23,7 & 26,3 & 13,2 & 36,8 & 76,3 & \\
\hline \multicolumn{7}{|l|}{ Hacinamiento } \\
\hline No hacinado & 42,4 & 38,4 & 11,2 & 8.0 & 57,6 & \multirow{3}{*}{0,000} \\
\hline Hacinamiento & 26,2 & 42,6 & 18,5 & 12,7 & 73,8 & \\
\hline Hacinamiento critico & 18,5 & 39,4 & 23,7 & 18,4 & 81,5 & \\
\hline \multicolumn{7}{|l|}{ Sexo del jefe del hogar } \\
\hline Hombre & 39,5 & 397 & 124 & 8.4 & 60.5 & \multirow{2}{*}{0,000} \\
\hline Mujer & 36,2 & 37,1 & 14,8 & 119 & 63,8 & \\
\hline \multicolumn{7}{|c|}{ Nivel educativo más alto del jefe del hogar } \\
\hline Ninguno & 21,6 & 37,2 & 19,7 & 21,4 & 78,4 & \multirow{10}{*}{0,000} \\
\hline Preescolar & 57,1 & 14,3 & 28.6 & 0,0 & 42,9 & \\
\hline Básica primaria & 31,7 & 42,1 & 15,7 & 10,5 & 68,3 & \\
\hline Básica secundaria y media & 42,8 & 39,7 & 10,9 & 6.5 & 57,2 & \\
\hline Técnico & 55,3 & 35,8 & 7,1 & 1.9 & 44,7 & \\
\hline Tecnológico & 67,5 & 26,9 & 4,4 & 1,3 & 32,5 & \\
\hline Universitaria sin título & 60,5 & 27,2 & 7,4 & 4,9 & 39,5 & \\
\hline Universitaria con título & 72,3 & 23,8 & 1,7 & 2,1 & 27,7 & \\
\hline Postgrado sin título & 80,0 & 20,0 & 0,0 & 0.0 & 20,0 & \\
\hline Postgrado con título & 82,1 & 17,1 & 0,4 & 0,4 & 17,9 & \\
\hline \multicolumn{7}{|c|}{ Actividad en la semana anterior a la entrevista } \\
\hline Buscando trabajo & 17,6 & 40,6 & 18,4 & 23,4 & 82,4 & \multirow{6}{*}{0,000} \\
\hline Oficios del hogar & 32,0 & 39,7 & 15,8 & 12,5 & 68,0 & \\
\hline Incapacitado permanente & 36,7 & 36,5 & 14,1 & 12,7 & 63,3 & \\
\hline Trabajando & 40,5 & 39,1 & 12,4 & 8,0 & 59,5 & \\
\hline Otra actividad & 44,4 & 35,8 & 11,9 & 7,8 & 55,6 & \\
\hline $\begin{array}{l}\text { Estudiando } \\
\text { - }\end{array}$ & 51,4 & 33,3 & 6,9 & 8,3 & 48,6 & \\
\hline
\end{tabular}


Estratificación de los hogares y seguridad alimentaria

Según el estrato por facturación de servicios públicos, a medida que ascendió el estrato, disminuyó la proporción de hogares en ISA. Esta misma tendencia se observó para todos los grados de ISA $(\mathrm{p}=0,000)$ (Tabla 2$)$.

Características de las viviendas y acceso a servicios públicos básicos El 27,4 \% y el 31,7\% de los hogares que vivían en cuartos en inquilinato $\mathrm{y}$ en otro tipo de cuarto, estaban en ISA moderada y severa respectivamente. Por cada 5 y 4,5 hogares hacinados y en ISA moderada y severa se presentó un hogar no hacinado respectivamente (Tabla 2).

Algunas características socio demográficas y de fuerza laboral La proporción de hogares en ISA fue superior en los hogares encabezados por mujeres $(\mathrm{p}=0,000)$. Según el nivel educativo del jefe del hogar a medida que éste asciende se disminuyó la proporción de ISA $(\mathrm{p}=0,000)$. Con respecto a la actividad realizada la semana anterior a la entrevista, se encontró la mayor proporción de hogares inseguros cuando el jefe del hogar estuvo buscando trabajo $(\mathrm{p}=0,000)$ (Tabla 2$)$.

Análisis multivariado

En cuanto a la prevalencia de ISA en los hogares, se halló que los hogares que cocinaban en un cuarto usado también para dormir, presentaron más posibilidad de inseguridad alimentaria que aquellos hogares que cocinaban en un cuarto usado solamente para cocinar. Asimismo, hogares que cocinaban con carbón mineral, presentaron una mayor probabilidad de experimentar inseguridad alimentaria, llama la atención como hogares con conexión a red de gas también mostraron mayor posibilidad de inseguridad (Tabla $3 a$ y $3 b$ ).

Se encontró que los hogares con casa propia pero que la estaban pagando o los que tenían posesión de la vivienda pero sin título, mostraron mayor probabilidad de experimentar inseguridad alimentaria, asimismo aquellos que manifestaron que los ingresos solo alcanzaban para cubrir los gastos mínimos ó los hogares en donde los ingresos no alcanzan para cubrir los gastos mínimos, presentando estos últimos 5,5 veces más posibilidad de experimentar inseguridad que los hogares en los que sus ingresos alcanzaban a cubrir más de los gastos mínimos. Además, los hogares en los que por falta de dinero algún integrante no consumió ninguna de las tres comidas aumentaron la posibilidad de inseguridad alimentaria 18,4 veces $(\mathrm{p}=0,0000)$, hallando también una mayor probabilidad de ISA a medida que el hogar presentó más nivel de hacinamiento. De igual manera, aspectos como perder el empleo por parte del jefe del hogar, atrasarse 
en el pago de los servicios públicos 4 meses ó más, disminuir el gasto en alimentación y el que el hogar se haya gastado los ahorros, mostraron un aumento en la probabilidad de ISA. Asimismo, hogares que manifestaron sentirse pobres, mostraron una probabilidad $0.44(\mathrm{p}=0,0000)$ más alta de experimentar ISA, a medida que descendió la percepción de las condiciones de vida del hogar, aumentó la probabilidad de ISA (Tabla 3a y 3b).

Tabla 3a. Razones de disparidad para la inseguridad alimentaria. Colombia, ECV 2008

\begin{tabular}{|c|c|c|c|c|c|}
\hline \multirow[t]{2}{*}{ Características } & \multirow[t]{2}{*}{$\mathrm{B}$} & \multirow[t]{2}{*}{$p$} & \multirow[t]{2}{*}{ OR } & \multicolumn{2}{|c|}{ I.C. $95 \%$} \\
\hline & & & & \multicolumn{2}{|c|}{ Lim. Inf Lim.Sup } \\
\hline \multicolumn{6}{|l|}{ Tipo de vivienda } \\
\hline Casa & & & 1,00 & & \\
\hline Apartamento & 0,00 & 0,9656 & 1,00 & 0,89 & 1,12 \\
\hline Cuartos en inquilinato & $-0,13$ & 0,5446 & 0,88 & 0,59 & 1,33 \\
\hline Cuartos en otro tipo de estructura & 0,24 & 0,1960 & 1,28 & 0,88 & 1,85 \\
\hline Otro tipo de vivienda & $-0,83$ & 0,1016 & 0,43 & 0,16 & 1,18 \\
\hline No tiene alcantarillado & 0,23 & 0,0001 & 1,26 & 1,12 & 1,42 \\
\hline \multicolumn{6}{|l|}{ Sitio preparación de alimentos } \\
\hline En un cuarto usado solo para cocinar & & & 1,00 & & \\
\hline En un cuarto usado también para dormir & 0,34 & 0,0315 & 1,40 & 1,03 & 1,91 \\
\hline En una sala-comedor con lavaplatos & 0,31 & 0,0700 & 1,36 & 0,98 & 1,90 \\
\hline En una sala-comedor sin lavaplatos & 0,39 & 0,0566 & 1,48 & 0,99 & 2,23 \\
\hline En un patio, corredor, enrramada, al aire libre & $-0,04$ & 0,7865 & 0,96 & 0,74 & 1,26 \\
\hline \multicolumn{6}{|c|}{ Combustible para cocinar } \\
\hline Electricidad & & & 1,00 & & \\
\hline Gas natural conectado a red pública & 0,35 & 0,0027 & 1,42 & 1,13 & 1,78 \\
\hline Petróleo, gasolina, kerosene, alcohol, cocinol & 0,13 & 0,2353 & 1,14 & 0,92 & 1,43 \\
\hline Gas propano (cilindro o pipeta) & 0,70 & 0,1562 & 2,01 & 0,77 & 5,29 \\
\hline Carbón mineral & 0,28 & 0,0282 & 1,32 & 1,03 & 1,69 \\
\hline Leña, madera o carbón de leña & 0,44 & 0,4719 & 1,56 & 0,47 & 5,22 \\
\hline \multicolumn{6}{|l|}{ Tenencia de la vivienda } \\
\hline Propia (totalmente pagada) & & & 1,00 & & \\
\hline Propia (la están pagando) & 0,38 & 0,0005 & 1,47 & 1,18 & 1,82 \\
\hline En arriendo o subarriendo & $-0,01$ & 0,8077 & 0,99 & 0,88 & 1,11 \\
\hline En usufructo & $-0,04$ & 0,4898 & 0,96 & 0,84 & 1,08 \\
\hline Posesión sin título & 0,62 & 0,0000 & 1,86 & 1,39 & 2,48 \\
\hline \multicolumn{6}{|l|}{ Condiciones de vida } \\
\hline Muy buenas & & & 1,00 & & \\
\hline Buenas & 0,54 & 0,0000 & 1,71 & 1,40 & 2,10 \\
\hline Regulares & 1,59 & 0,0000 & 4,91 & 3,95 & 6,10 \\
\hline Malas & 2,32 & 0,0000 & 10,16 & 5,71 & 18,05 \\
\hline \multicolumn{6}{|l|}{ Percepción pobreza } \\
\hline $\mathrm{Si}$ & 0,37 & 0,0000 & 1,44 & 1,31 & 1,59 \\
\hline \multicolumn{6}{|l|}{ Ingresos del hogar } \\
\hline Cubren más que los gastos minimos & & & 1,00 & & \\
\hline No alcanzan para cubrir los gastos mínimos & 1,88 & 0,0000 & 6,54 & 5,42 & 7,87 \\
\hline Sólo alcanzan para cubrir los gastos mínimos & 0,94 & 0,0000 & 2,57 & 2,17 & 3,05 \\
\hline \multicolumn{6}{|c|}{ Por falta dinero no consumió tres comidas un miembro hogar } \\
\hline $\mathrm{Si}$ & 2,96 & 0,0000 & 19,39 & 10,86 & 34,63 \\
\hline \multicolumn{6}{|l|}{ Hacinamiento } \\
\hline Sin hacinamiento & & & 1,00 & & \\
\hline Hacinamiento & 0,31 & 0,0000 & 1,36 & 1,18 & 1,57 \\
\hline Hacinamiento crítico & 0,42 & 0,0000 & 1,52 & 1,27 & 1,82 \\
\hline
\end{tabular}




\section{DISCUSIÓN}

Los hogares Colombianos que participaron en la ECV del año 2008, presentan una prevalencia de inseguridad alimentaria $(58,2 \%)$ superior a las reportadas para los hogares de Colombia por la ENSIN-2005, (40,6 \% IC: 38,7-42,4 \%) (6). El incremento de la prevalencia de inseguridad alimentaria obedece en parte porque en la ECV, se empleó la Escala Latinoamericana para la Medición de la Seguridad Alimentaria en América Latina (13), adaptada y validada por Álvarez $(11,12)$, la cual utilizó un filtro para la clasificación más exigente al empleado en la ENSIN $2005(6,14)$. Aunque se debe considerar los efectos de la profundización de la pobreza y el incremento del precio de los alimentos. En Colombia, durante el período 2005 al 2008 la población en indigencia pasó de 15,7 a 17,8 \% (15), y del año 2005 al 2008 la inflación de los alimentos en el grupo de ingresos más bajos estuvo por encima de la inflación total (15), con el agravante que entre 2007 y 2008 , los precios de los alimentos esenciales para la canasta básica de alimentos: arroz, trigo, y maíz se incrementaron entre 80 y $90 \%$ (16).

Tabla 3b. Razones de disparidad para la inseguridad alimentaria.

\begin{tabular}{|c|c|c|c|c|c|}
\hline \multirow{2}{*}{ Caracteristicas } & \multirow[t]{2}{*}{$\mathrm{B}$} & \multirow[t]{2}{*}{$p$} & \multirow[t]{2}{*}{ OR } & \multicolumn{2}{|c|}{ I.C. $95 \%$} \\
\hline & & & & \multicolumn{2}{|c|}{ Lim. Inf Lim.Sup } \\
\hline & & & & & \\
\hline Postgrado con titulo & & & 1,00 & & \\
\hline Ninguno & 1,15 & 0,0000 & 3,17 & 2,09 & 4,81 \\
\hline Preescolar & 0,82 & 0,3380 & 2,26 & 0,43 & 11,97 \\
\hline Básica primaria & 0,95 & 0,0000 & 2,58 & 1,75 & 3,81 \\
\hline Básica secundaria y media & 0,86 & 0,0000 & 2,36 & 1,60 & 3,48 \\
\hline Técnico & 0,82 & 0,0003 & 2,27 & 1,46 & 3,53 \\
\hline Tecnológico & 0,30 & 0,2839 & 1,34 & 0,78 & 2,31 \\
\hline Universitaria sin título & 0,42 & 0,1308 & 1,52 & 0,88 & 2,60 \\
\hline Universitaria con titulo & 0,36 & 0,1052 & 1,43 & 0,93 & 2,21 \\
\hline Postgrado sin título & 0,09 & 0,9044 & 1,09 & 0,26 & 4,60 \\
\hline Ocupación jefe hogar (la semana anterior) & & & & & \\
\hline Trabajando & & & 1,00 & & \\
\hline Buscando trabajo & 0,37 & 0,0162 & 1,45 & 1,07 & 1,96 \\
\hline Estudiando & 0,86 & 0,3362 & 2,36 & 0,41 & 13,50 \\
\hline Oficios del hogar & 0,16 & 0,0123 & 1,17 & 1,03 & 1,32 \\
\hline Incapacitado permanente para trabajar & $-0,17$ & 0,2029 & 0,84 & 0,65 & 1,10 \\
\hline Otra actividad & $-0,04$ & 0,6940 & 0,96 & 0,79 & 1,17 \\
\hline $\begin{array}{l}\text { Pérdida de empleo jefe hogar } \\
\mathrm{Si}\end{array}$ & 0,47 & 0,0000 & 1,61 & 1,35 & 1,92 \\
\hline $\begin{array}{l}\text { Atraso pago servicios públicos } \approx 4 \text { meses o más } \\
\mathrm{Si}\end{array}$ & 0,42 & 0,0010 & 1,52 & 1,19 & 1,96 \\
\hline $\begin{array}{l}\text { Uno o más miembro empezaron a trabajar } \\
\text { Si }\end{array}$ & 0,34 & 0,0061 & 1,41 & 1,10 & 1,80 \\
\hline $\begin{array}{l}\text { Disminución gasto alimentario } \\
\mathrm{Si}\end{array}$ & 0,53 & 0,0001 & 1,69 & 1,31 & 2,18 \\
\hline $\begin{array}{l}\text { Endeudamiento hogar } \\
\text { Si }\end{array}$ & 0,52 & 0,0000 & 1,68 & 1,40 & 2,03 \\
\hline
\end{tabular}


Los hogares rurales presentaron una prevalencia ISA, mayor a la encontrada en la zona urbana, igual situación se reporta en diversos estudios (6, 17-19). Al respecto la CEPAL, plantea que las personas más vulnerables viven en el área rural o en zonas periféricas a las cabeceras municipales, tienen poco acceso a los servicios de agua potable y saneamiento, presentan bajo nivel educativo y son herederos de condiciones socioeconómicas desfavorables (20).

La prevalencia de ISA decrece a medida que los hogares ascienden en su estrato socioeconómico. Los clasificados en estrato bajo-bajo reportaron prevalencias de inseguridad alimentaria moderada y severa muy elevadas, lo que implica que alguno(s) de los miembros del hogar hayan tenido hambre, cuyos efectos acarrea sufrimientos psicológicos y perturbaciones sociofamiliares en los hogares que la padecen (21). El hambre interfiere de manera muy importante en el alcance de las capacidades básicas para el adecuado funcionamiento de las personas (22).

Los hogares que poseen viviendas sin título, reportan mayor prevalencia de ISA severa, situación ilegal a la cual se ven abocados los hogares pobres dado que a la legalidad sólo se accede mediante el poder económico y político del cual carecen (23). Igual situación se presenta en los hogares hacinados y en hacinamiento crítico, situación que agrava la condición nutricional, dado que los integrantes del hogar están más vulnerables a adquirir enfermedades infecciosas (24).

Los hogares encabezados por mujeres, presentaron una proporción de ISA mayor que en aquellos encabezados por hombres, situación que se puede explicar en parte porque el bajo capital humano de las mujeres de los hogares en ISA, limita la inserción laboral, y la responsabilidad con los hijos se convierte en una barrera para entrar en el mercado de trabajo (25). En lo concerniente al género en la determinación de los salarios, un informe de la Cepal mostró que en todos los países incluidos en el estudio, las mujeres percibían menores ingresos salariales que los varones, aunque tuvieran igual nivel de escolaridad y experiencia laboral (26).

Las mujeres jefe de hogar, en muchas ocasiones presentan menor educación y menor acceso a diferentes recursos, situación que le impone algunas restricciones en su desarrollo, ya sea porque enfrenten una discriminación en el mercado laboral o porque las obligaciones del trabajo doméstico y el cuidado de los hijos las obligue a escoger trabajos que se 
adecuen a sus responsabilidades, pero en muchas ocasiones estos trabajos cuentan con una menor remuneración (27-29).

A menor nivel educativo del jefe de hogar, se presenta mayor prevalencia de inseguridad alimentaria, esta misma situación es reportada en la ENSIN2005 (6). El nivel educativo de los jefes de hogar constituye una de las principales dotaciones de la familia para lograr mejores ingresos económicos e incrementar el acceso de los alimentos en el hogar. En América Latina, la educación explica en promedio el $38 \%$ de las variaciones en los salarios urbanos y el $52 \%$ en Colombia, es decir, es el factor que más contribuye a la desigualdad salarial en los países de la región $(30,31)$. Sen plantea que entre mayor sea la cobertura de la educación básica, incluso las personas potencialmente pobres, tienen más oportunidad de vencer la miseria (22).

La pobreza subjetiva revela justamente lo que sucede con las percepciones de las personas en cuanto a su condición de ingresos, variable que es considerada como el elemento central en la posibilidad de alcanzar o mejorar su bienestar económico, entendiendo por bienestar económico justamente a aquella parte del bienestar que está ligada a los ingresos monetarios, como es el bienestar nutricional (32). Aunque, en las tres últimas décadas se advierte claramente que los análisis de pobreza tienden a adoptar un enfoque más "holístico", que consiste en pasar de un enfoque estrecho y estático, concentrado en los ingresos y el consumo, al reconocimiento de la pobreza como un fenómeno multidimensional que, además de tener aristas relacionadas con la privación física, comprende factores no materiales que se vinculan con la privación social, como la autoestima, el respeto, el poder y la vulnerabilidad (33). La anterior postura es coherente con el concepto de calidad de vida, el cual está relacionado con el bienestar social y depende de la satisfacción de las necesidades humanas y de los derechos positivos (22), de los modos de vida, del trabajo, de servicios sociales y condiciones ecológicas.

ELCSA, por primera vez se aplica en una muestra nacional de hogares, razón por la cual con este instrumento no se puede analizar las tendencias de la seguridad alimentaria de Colombia, ni sus resultados se pueden comparar con los arrojados por la Encuesta Nacional de Nutrición ENSIN 2005, debido a que ésta utilizó otra escala

Agradecimientos: Los investigadores agradecen al DANE y al ICBF, quienes propiciaron que ELCSA fuera incluida en la ECV del 2008, a la FAO quien apoyó la 
investigación y a la Universidad de Antioquia quien mediante la estrategia de sostenibilidad 2009-2010, auspicio la elaboración de éste artículo.

\section{REFERENCIAS}

1. FAO. Cumbre Mundial Sobre la Alimentación. Roma; 1996.

2. Pelletier $D$, Olso $C$, Fronguillo E. Inseguridad alimentaria hambre y desnutrición. En: Bowman BA, Russel RM. Conceptos Actuales sobre Nutrición. Octava edición ed. Washington; Publicación científica de la OPS/OMS. 2003.

3. Radimer K, Olson C, Greene J, Campbell C, Habicht J. Understanding hunger and developing indicators to assess it in women and children. J Nutr Educ. 1992; 24:36S-45S.

4. Wehler $\mathrm{C}$, Scott R, Andeson J. The community childhood identification project: a model of domestic hunger. J Nutr. 1992; 24:29S-35S.

5. Frongillo EA, Jr. Validation of measures of food insecurity and hunger. J Nutr. 1999; 129(2S Suppl):506S-509S.

6. Álvarez M, Estrada A. Evaluación cualitativa de la seguridad alimentaria en el hogar. En: Encuesta nacional de la situación nutricional de Colombia, ENSIN 2005. Bogotá: ICBF; 2005. p. 322-331.

7. Álvarez M, López A, Monsalve J, Giraldo N, Zapata O, Vélez O, et al. Contexto sociodemográfico, estado nutricional y de salud e ingesta dietética de los niños que participan del programa de Complementación Alimentaria alianza MANA ICBF. Evaluación económica, de la participación comunitaria e institucional para el desarrollo del programa y utilización del complemento alimentario en el hogar. Medellín; 2007.

8. Oshaug A, Eide W. Food security and the right to food in international law and development. New York: UNICEF; 1992.

9. Dehollaín P. Conceptos y factores condicionantes de la seguridad alimentaria en hogares. Arch Latinoamericanos de Nutrición. 1995;45(1):338-40.

10. DANE. Encuesta de Calidad de vida en Colombia 2008. Bogotá; 2008.

11. Álvarez M, Vélez O. Componente adaptación lingüística de ELCSA. Medellín: ICBF, PMA,FAO,DANE,UDEA.; 2008.

12. Álvarez M, Estrada A, Melgar-Quiñonez H, Fonseca Z. Adaptación y validación interna y externa de la Escala Latinoamericana y Caribeña para la medición de seguridad alimentaria en el hogar - ELCSA- Colombia: Componente adaptación lingüística de ELCSA. Medellín: ICBF, PMA, FAO, DANE, UDEA.; 2008.

13. Pérez-Escamilla R, Melgar-Quiñonez $H$, Nord M, Álvarez M, Segall-Correa AM. Escala Latinoamericana y Caribeña de Seguridad Alimentaria -ELCSA-. Perspectivas en Nutrición Humana 2007; Separata: 117-134.

14. Álvarez M, Estrada A, Montoya E, Melgar-Quiñonez H. Validación de escala de la seguridad alimentaria doméstica en Antioquia, Colombia. Rev Salud Pública de México. 2006; 48(6):474-481.

15. República de Colombia. Departamento Nacional de Planeación [Internet]. Pobreza por ingreso autónomo y distribución del ingreso 2009. http://www.dnp.gov.co/ PortalWeb/LinkClick.aspx. Consultado febrero de 2010.

16. Hopenhayn M, Beccaria L, Feres JC, Jaspers_Faijer D, Arriagada I, Badilla M, Cecchini S, Espindola E, Mancero X, Miranda F, Martinez C, Miller T, Saad Py, Villatoro P. Pobreza, exclusión social y desigualdad distributiva. En: Hopenhayn M, Beccaria L, Feres JC, Jaspers_FD, Arriagada I, Badilla M, Cecchini S, Espindola E, Mancero X, Miranda F, Martínez C, Miller T, Saad PY, Villatoro P. Panorama Social de América Latina 2008. Santiago de Chile: Naciones Unidas: 2009.

17. Álvarez M, Benjumea M, Roldán P, Maya M, Martínez M, Montoya E. Perfil Alimentario y Nutricional de los Hogares de Antioquia. Medellín: Divergráficas. S.A; 2005. 
18. Álvarez M, López A, Monsalve J, Restrepo A, Botero J, Forero Y. Contexto socioeconómico de los niños que participan en el programa de complementación alimentaria alianza MANA-ICBF. En: Álvarez MC, editor. Contexto socioeconómico, estado nutricional y de salud e ingesta dietética de los niños que participan en el programa de complementación alimentaria alianza MANA-ICBF. Medellín; 2006. p. 31-129.

19. Segall-Correa AM, Panigassi G, Sampaio Md Mde F, Marin-Leon L, Pérez-Escamilla R. Validación de un instrumento de inseguridad alimentaria y hambre en el contexto de la política brasilera de combate al hambre. Perspectivas en Nutrición Humana. 2007; Separata:89-102.

20. Martínez R, León A, Espíndola E, Fernández A, Ávila A, Pinto P. Hambre y desigualdad en los países andinos. La desnutrición y la vulnerabilidad alimentaria en Bolivia, Colombia, Ecuador y Perú. Santiago: Naciones Unidas; 2005. p. 10-12.

21. Webb P, Coates J, Frongillo EA, Rogers BL, Swindale A, Bilinsky P. Measuring household food insecurity: why it's so important and yet so difficult to do. J Nutr. 2006; 136(5):1404S-1408S.

22. Sen A. La pobreza como privación de capacidades. En: Sen A. Desarrollo y libertades. Santa Fe de Bogotá: Planeta Colombiana Editorial S.A.; 2000.

23. Salas J. Latinoamérica: Hambre de vivienda. Santiago de Chile: Universidad de Chile; 2002. 59-68.

24. Hernández F, Rodríguez Z, Herrera FNT. Enfermedad diarreica aguda en niños: comportamiento de algunos factores de riesgo. Revista Cubana de Medicina General Integral. 2000; 16:129-33.

25. Trejos J, Montiel N. El capital de los pobres en Costa Rica: acceso, utilización y rendimiento. El trimestre económico. 1999; 65(3):553-618.

26. Godoy L. Entender la pobreza desde la perspectiva de género. Santiago: Naciones Unidas; 2004. p. 23-25.

27. Acosta F. Jefatura de hogar femenina y bienestar familiar: resultados de la investigación empírica. Papeles de población. 2001; 28:41-97.

28. CEPAL. Ciudadanía, igualdad y cohesión social: la ecuación pendiente. En: CEPAL. Equidad, desarrollo y ciudadanía. Santiago de Chile: Naciones Unidas; 2000.

29. Artiles L[Internet]. Pobreza y género. Rev Cubana Salud Pública.2007;33(4). Disponible en: http://bvs.sld.cu/revistas/spu/vol33_4_07/spu04407.html. Consultado febrero de 2010.

30. CEPAL. Panorama social de América Latina 2006. Santiago de Chile; 2007.

31. Perry G, Arias O, López J, Maloney W, Servén L. Reducción de la pobreza y el crecimiento: Círculo virtuosos y círculo vicioso. Resumen ejecutivo: Banco Mundial; 2006.

32. Giarrizzo V. Percepciones de Pobreza y Pobreza Subjetiva Un estudio para la Argentina. Buenos Aires: Universidad de Buenos Aires; 2007.

33. Chant S. La incorporación de la perspectiva de género en el análisis de la pobreza: contribuciones feministas a la conceptualización y medición de la pobreza, 19702000. En: Chant S. Nuevas contribuciones al análisis de la pobreza: desafíos metodológicos y conceptuales para entender la pobreza desde una perspectiva de género. Santiago de Chile: Naciones Unidas; 2003. 